16 a 18 de outubro de 2019 - Campinas | Brasil

\title{
Uterine Sarcomas attended at Brazilian Referral Hospital: a 16-year study
}

\section{Melissa L. M. Silva*, Julio C. Teixeira.}

\begin{abstract}
Uterine sarcomas are unusual pelvic neoplasms, accounting for $5 \%$ of cancers of the uterine corpus and their incidence are increasing with the aging of the population. This study aims to assess the pattern of uterine sarcomas diagnosis related to symptons and method for diagnosis, year, age, stage and histological types in the period from 2001 to 2016. There were 123 sarcomas, a higher proportion ( 10\%) of all uterine corpus neoplasms. The initial signs or symptoms were abnormal uterine bleeding in $77 \%$, and main diagnosis method were uterine curettage $(35 \%)$ or surgery $(34 \%)$. A increasing trend of uterine sarcoma with +1.2 cases every 2 years were observed $(p<0.05)$. Diagnosis were done in $62 \%$ of women with age $>60 \mathrm{yo}$ and $56 \%$ in advanced stage II-IV. Histology: $47 \%$ carcinosarcomas, $22 \%$ leiomyosarcomas, $16 \%$ endometrial stromal sarcomas (ESS), and 14\% adenosarcomas. $76 \%$ of carcinosarcomas were diagnosed in age $>=60$ yo and $58 \%$ in stage III or IV, while $59 \%$ leiomyosarcomas and 50\% ESS were registered in <60yo and $56 \%$ and $80 \%$ in stage I-II, respectively. Conclusion: Uterine sarcomas rates increased in the period 2001-2016, prevailing in age of 60 years or more and $56 \%$ at advanced stage, with predominance of carcinosarcomas and leiomyosarcomas.
\end{abstract}

Key words: sarcoma, corpus uterine, epidemiology.

\section{Introduction}

Corpus uterine neoplasms are compound by $95 \%$ of carcinomas and $5 \%$ of sarcomas. Sarcomas of the uterus are tumors arising from proliferation of mesenchymal cells and have a diversity of histologic types, wich makes it difficult to study. Uterine sarcomas usually are diagnosed in advanced stage with aggressive behavior and early distant metastases.

Women's Hospital at University of Campinas, Campinas, $\mathrm{SP}$, Brazil, is a regional Cancer Center, part of Brazilian Public Health System (SUS), and care women with gynecologic cancer. There is a Hospital-Based Cancer Registry (HCR) system since 2000, with consistent information used by Brazilian National Cancer Institute (INCA) to produce official estimates.

This is an observational study that aims to describe the evolutionary pattern of diagnosis of uterine sarcomas in the period 2001 to 2016, regard signs or symptoms related to initial diagnosis, method for diagnosis, year, age, histologic types and stage in diagnosis. All ethical principles will be fulfilled in this work. All women will have their data and medical records kept under confidentiality.

\section{Results and Discussion}

There were 1,191 neoplasms of uterine corpus recorded in HCR between 2001-2016 with 123 sarcomas of the uterus, a higher proportion ( 10\%) than expected (Figure 1).

The initial signs or symptoms were abnormal uterine bleeding in $77 \%$, abnormal image exam in $7 \%$, increased abdominal volume in $6 \%$, pelvic pain in $4 \%$, and a vaginal birth tumor in $3 \%$. Diagnosis methods were uterine curettage $(35 \%)$, surgery $(34 \%)$, hysteroscopy $(13 \%)$, and biopsy at vaginal examination in $17 \%$.

Figure 1. Distribution of 1,191 neoplasms of uterine corpus recorded in period 2001-2016.

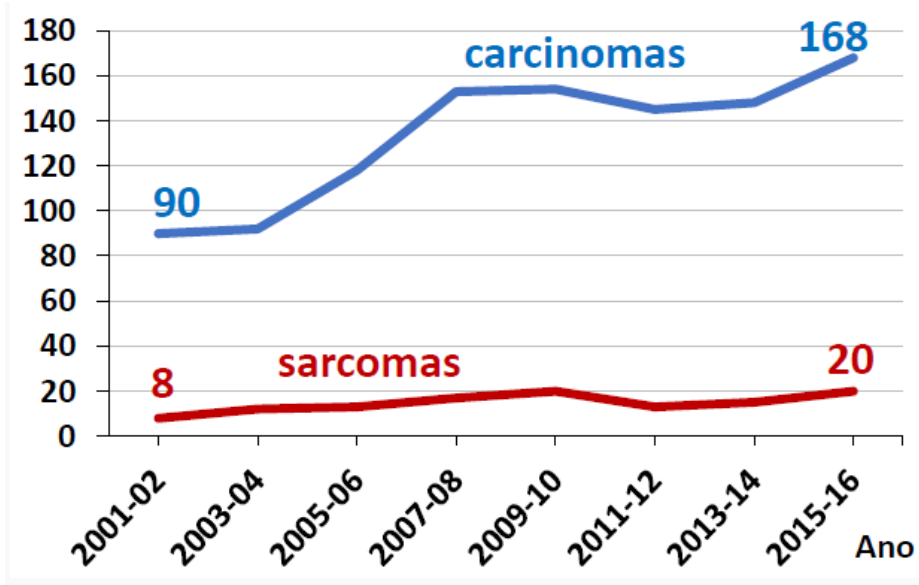

There were 16 cases of sarcomas diagnosed by hysterectomy due to myomatosis, all in women aged up to 55 years.

Analyzes done by Chi square trend test exhibited a significant increasing trend of uterine sarcoma diagnosis (8 cases in 2001-2002 vs. 20 in 2015-2016) with +1.2 cases every 2 years $(p<0.05$; Figure 1$)$. Diagnosis were done in $62 \%$ of women with age $\geq 60$ yo and $56 \%$ in advanced stage II-IV. Histology: $47 \%$ carcinosarcomas, $22 \%$ leiomyosarcomas, $16 \%$ endometrial stromal sarcomas (ESS), and $14 \%$ adenosarcomas. $76 \%$ of carcinosarcomas were diagnosed in age >=60yo and $58 \%$ in stage III or IV while 59\% leiomyosarcomas and 50\% ESS were registered in $<60$ yo and $56 \%$ and $80 \%$ in stage I-II, respectively.

\section{Conclusions}

Uterine sarcomas rates increased in the period 2001 2016 , prevailing in age of 60 years or more and $56 \%$ at advanced stage, with predominance of carcinosarcomas and leiomyosarcomas 